Session: Industrial applications of hybrid RANS-LES methods

\title{
Hybrid RANS/LES of an isolated engine nacelle with side wind using an unstructured CFD solver
}

\author{
Marco Burnazzi*, Axel Probst*, Mathias Steger** \\ * Institute of Aerodynamics and Flow Technology, DLR Göttingen \\ ** Design Systems Engineering, Aerodynamics \& Aeroacoustics, \\ Rolls-Royce Deutschland
}

\begin{abstract}
The present work focuses on the scale-resolving numerical simulation of an isolated jet-engine intake in presence of side wind. The computation was carried out with the DLR TAU code within the framework of the Rolls-Royce funded project FaNcI (Fan-Nacelle Integration) and relied on advanced numerical techniques recently developed as part of the DLR project VicToria (Virtual Aircraft Technology Integration Platform)[1]. The FaNcI project aims at enhancing the accuracy of numerical and experimental prediction techniques for the analysis of side wind effects on installed and isolated jet engines. The configuration investigated here reproduced an experimental set-up where an engine nacelle was mounted perpendicular to the side wall of a pressurized wind tunnel test section and was used as one of the test cases in the EU project ATAAC [2]. The wind tunnel fan generated the side wind whereas blowing air from the test section to ambient through the intake model simulated the engine operation. The combination of flow blowing through the engine intake and side wind caused a shock-induced flow separation on the upstream region of the nacelle lip. The goal of the present work was the simulation of the flow in off-design condition with high crosswind: a partially separated flow where the flow reattachment takes place in the proximity of the hypothetical fan location. Under these conditions, an accurate numerical prediction of the flow topology and of the total pressure losses inside the engine intake requires a turbulence model able to account for unsteady flow behavior on a broad range of temporal and spatial scales, high streamline curvature and turbulence anisotropy. Such a high flow complexity calls for the resolution of turbulent structures following an LES-like approach. In particular, a hybrid RANS/LES method based on the Improved Delayed Detached-Eddy Simulation (IDDES) was applied here. This approach distinguishes between attached flow, to be treated in RANS mode, and detached LES regions. Moreover, it also provides wallmodeled LES capabilities in resolved flow after reattachment, which is
\end{abstract}


Session: Industrial applications of hybrid RANS-LES methods

considered crucial to model the complex 3-D separation patterns in engine inlets correctly. As RANS background, the Menter-SST model was used.

To provide sufficient numerical accuracy for scale-resolving simulations (such as IDDES), the low-dissipation low-dispersion (LD2) scheme was applied [3]. This scheme combines a skew-symmetric central discretization of the spatial fluxes, a shock-adapted mix of 2nd- and 4thorder artificial matrix dissipation, and a low-dispersive reconstruction of the face states. To ensure robustness on complex grid topologies and flow phenomena involving strong gradients, the scheme is blended with a standard central discretization using a suitable blending approach for hybrid RANS/LES, i.e. the LD2 scheme is mainly active in LES regions.

The analysis of the numerical results focuses on the differences between the IDDES and the RANS simulations, the resolution of threedimensional turbulent structures (see Figure 1) as well as the insight into the flow physics yielded by the scale-resolving simulation. Finally, the result validation is based on experimental measurements of the flow distortion inside the engine inlet. From the final picture the need for scaleresolving turbulence simulation and the advantage of a specific numerical scheme clearly emerge.

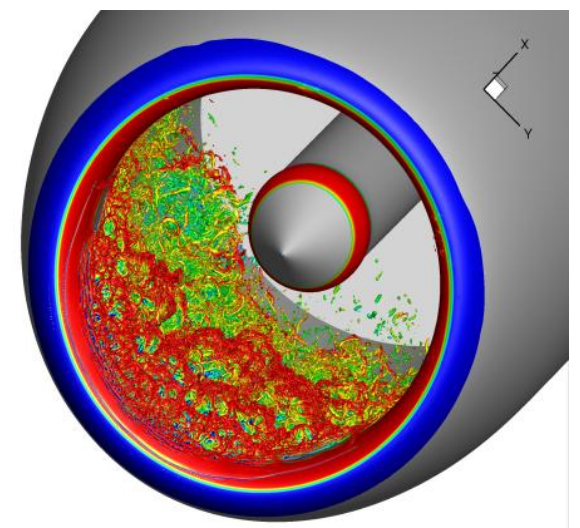

Fig. 1: Resolved turbulence inside the nacelle intake

\section{References}

[1] Görtz, S.: "Projektplan, VicToria (Virtual Aircraft Technology Integration Platform)", Duration: 01.07.2016 - 31.12.2019, Project leader: Dr. S. Görtz, Institute of Aerodynamics and Flow Technology, DLR Braunschweig.

[2] Schwamborn, D.: "Results and Lessons learned from the EU-Project ATAAC", ERCOFTAC 2013.

[3] Probst, A., Löwe, J., Probst, S., Knopp, T., and Kessler, R.: "Scale-Resolving Simulations with a Low-Dissipation Low-Dispersion Second-Order Scheme for Unstructured Finite-Volume Flow Solvers”, AIAA SciTech, 2015. 